\title{
Retrospective Analysis of Hysterectomy Cases in a Tertiary Care Institute, JNIMS
}

\author{
Mayanglambam Ronita Devi ${ }^{1}$, Helen Kamei ${ }^{2}$, Madhuri Laishram ${ }^{3}$, Umalakshmi Lairellakpam ${ }^{4}$, Kimthianmuang Guite ${ }^{5}$, \\ Ishor Kharel ${ }^{6}$
}

\begin{abstract}
Aim: To study the indications and clinical profile of patients undergoing hysterectomy in Jawaharlal Nehru Institute of Medical Sciences (JNIMS). Materials and methods: A chart review of 132 hysterectomy cases in JNIMS, from January 2016 to January 2017, was done. Peripartum hysterectomy was excluded. The data regarding age, parity, indications of hysterectomy, length of hospital stay (LOHS), and additional surgical procedures were collected and analyzed.

Results: Age distribution ranged from 34 to 73 years. The most common age-group was 41 to 50 years (57 patients, $43.18 \%$ ), and multiparas ( $>2$ parity) had maximal rate (68 patients, $51.52 \%$ ) of hysterectomies. The most common indication for hysterectomy was abnormal uterine bleeding (AUB) (56 patients, 4242\%). The abdominal route (75.76\%) was more common than the vaginal route (23.48\%) and minimal access surgery (MAS) (0.76\%). Eighty-seven patients (65.91\%) underwent bilateral salpingo-oophorectomy. The most noted frequency of hospital stay was 4 days (29 patients, 21.97\%). Eleven appendectomies and one cholecystectomy were done as an additional surgical procedure.

Conclusion: Nondescent vaginal hysterectomy (NDVH) is an established safe surgical procedure but such route is lesser used. The reason may be due to less exposure to such a procedure. Additional surgical procedure is an advantage in the abdominal route, but studies of the plausible risks that may be associated with such procedures are also lacking. The impact of preservation of the ovary is also a lesser-explored area. Hysterectomy seems to be the first option in treatment where resources are limited. It is time to review our approach to benign conditions before considering surgery as the first option.

Clinical significance: Symptomatic relief is palpable by hysterectomy, but lack of studies involving the impact of hysterectomy, for example, psychological, social, economical, etc., is a cause of concern. More prospective data comparing indications of hysterectomy with its impact may be helpful in streamlining absolute indications, and patients may be benefitted from the adverse effects of surgical interventions.

Keywords: Abnormal uterine bleeding, Fibroid, Hysterectomy, Indications, Nondescent vaginal hysterectomy.

Journal of South Asian Federation of Obstetrics and Gynaecology (2021): 10.5005/jp-journals-10006-1877
\end{abstract}

\section{INTRODUCTION}

Hysterectomy is done for benign and malignant cases. While definite indications hold true for malignant cases as curative, options of medical and conservative surgical procedures are available for benign cases. While symptomatic relief is appreciated, the surgical procedure itself carries the risk of complicationsimmediate and remote; it has socioeconomic and psychological implications too. Through our retrospective analysis, we aim to study the indications and clinical profile of patients undergoing hysterectomy in our institute.

\section{Materials and Methods}

A chart review was done of cases that had undergone a hysterectomy in Department of Obstetrics and Gynaecology of Jawaharlal Nehru Institute of Medical Sciences (JNIMS), Manipur, from January 2016 to January 2017. Peripartum hysterectomy cases were excluded from the study. Age, parity, complaints, indications of hysterectomy, types of hysterectomy, length of hospital stay (LOHS), and additional surgical procedures were collected and then analyzed.

\section{Results and Analysis}

A total of 132 hysterectomies were recorded from January 2016 to January 2017, that is, a span of 1 year and 1 month. The commonest age-group undergoing the procedure was 41 to 50 years group. There were 57 patients (43.18\%). These were followed by

\footnotetext{
${ }^{1-6}$ Department of Obstetrics and Gynaecology, Jawaharlal Nehru Institute of Medical Sciences, Imphal, Manipur, India

Corresponding Author: Helen Kamei, Department of Obstetrics and Gynaecology, Jawaharlal Nehru Institute of Medical Sciences, Imphal, Manipur, India, Phone: +91 9612910365, e-mail: helendrkamei75 @gmail.com

How to cite this article: Devi MR, Kamei $H$, Laishram $M$, et al. Retrospective Analysis of Hysterectomy Cases in a Tertiary Care Institute, JNIMS. J South Asian Feder Obst Gynae 2021;13(2):97-100.

Source of support: Nil

Conflict of interest: None
}

31 to 40 years age-group, and there were 37 patients (28.03\%) (Table 1). The mean age was $46.53 \pm 0.668$ years.

Multiparas (>para 2 group) had a maximal rate of hysterectomy, that is, 68 patients $(51.52 \%)$, and these were followed by parity 2 group, that is, 35 patients (26.51\%). Nulliparas had a minimal rate of hysterectomy, and there were 12 patients (9.09\%) (Table 1).

The most common indication for hysterectomy was abnormal uterine bleeding (AUB), that is, 56 patients (42.42\%), and these were followed by uterine fibroid, that is, 34 patients (25.76\%). With increasing age, the frequency for hysterectomy indication was noted to be due to genital prolapse, that is, 17 patients (12.12\%) (Table 2).

One hundred patients underwent hysterectomy by abdominal route $(75.76 \%)$. The types of hysterectomy done by abdominal 
routes were total abdominal hysterectomy (TAH), subtotal hysterectomy (sub-TAH), total hysterectomy with unilateral salpingo-oophorectomy (TAH + USO), and total hysterectomy with bilateral salpingo-oophorectomy (TAH + BSO). Thirty-one patients were done by vaginal route (24.24\%), and only one patient by minimal access surgery (MAS), that is, laparoscopy-assisted vaginal hysterectomy $(\mathrm{LAVH})(0.76 \%)$. The types of vaginal operation included nondescent vaginal hysterectomy (NDVH) and vaginal hysterectomy with pelvic floor repair (VH + PFR). We performed 14 NDVH (10.60\%) (Table 3).

Eighty-seven patients (65.91\%) underwent bilateral salpingooophorectomy, and while seven patients underwent unilateral salpingo-oophorectomy, the remaining 38 patients' ovaries were not removed. There was a tendency to retain bilateral ovaries in patients less than 35 years of age. Bilateral salpingo-oophorectomy was more frequent in 41 to 50 years age-group and above (Table 3 ).
The most noted frequency for LOHS was 4 days, and there were 29 patients (21.97\%). Fifteen patients with LOHS more than 10 days had preoperative morbidities like previous-cesarean delivery, anemia, hypertension, diabetes mellitus, etc. (Table 4). The mean LOHS was $6.07 \pm 0.376$ days. The most common indication in the abdominal approach was AUB, that is, 45 out of 100 patients, followed by uterine fibroids (34 patients). In the vaginal route, $\mathrm{VH}+\mathrm{PFR}$ was done in 17 patients with genital prolapse, and NDVH was done in 10 patients with AUB and 4 patients with chronic pelvic pain (CPP) (Table 5). Additional surgical procedures were noted for 12 patients (11 appendectomies and 1 cholecystectomy).

\section{Discussion}

In our study period of 1 year and 1 month, 132 patients underwent hysterectomy in our institute, JNIMS. The study showed

Table 1: Age-group-wise distribution with parity

\begin{tabular}{llllll}
\hline Age-group in years & Nulliparas & Para 1 & Para 2 & > Para 2 & Total \\
\hline $30-40$ & 3 & 7 & 12 & 15 & $37(28.03 \%)$ \\
$41-50$ & 7 & 7 & 13 & 30 & $57(43.18 \%)$ \\
$51-60$ & 2 & 2 & 9 & 19 & $32(24.24 \%)$ \\
$>61$ & - & 1 & 1 & 4 & $6(4.55 \%)$ \\
Total & $12(9.09 \%)$ & $17(12.88 \%)$ & $35(26.51 \%)$ & $68(51.52 \%)$ & $132(100 \%)$ \\
\hline
\end{tabular}

Table 2: Age-group-wise indications of hysterectomy

\begin{tabular}{|c|c|c|c|c|c|c|}
\hline Age-group in years & Fibroid & $A \cup B$ & Genital prolapse & $C P P$ & Adnexal SOL & Total \\
\hline $30-40$ & 13 & 14 & - & 7 & 3 & 37 (28.03\%) \\
\hline $41-50$ & 18 & 26 & 5 & 6 & 2 & $57(43.18 \%)$ \\
\hline $51-60$ & 3 & 16 & 7 & 3 & 3 & 32 (24.24\%) \\
\hline$>61$ & - & - & 5 & - & 1 & $6(4.55 \%)$ \\
\hline Total & 34 (25.76\%) & 56 (42.42\%) & 17 (12.88\%) & 16 (12.12\%) & 9 (6.82\%) & $132(100 \%)$ \\
\hline
\end{tabular}

AUB, abnormal uterine bleeding; CPP, chronic pelvic pain; Adnexal SOL, Adnexal space-occupying lesion

Table 3: Age-group-wise distribution of types of hysterectomy

\begin{tabular}{lllllllll}
\hline Age-group (years) & TAH & Sub-TAH & TAH+ USO & TAH+BSO & NDVH & VH + PFR & LAVH & Total \\
\hline $30-40$ & 4 & - & 6 & 21 & 5 & 1 & - & $37(28.03 \%)$ \\
$41-50$ & 1 & 1 & 1 & 43 & 6 & 4 & 1 & $57(43.18 \%)$ \\
$51-60$ & - & - & - & 22 & 3 & 7 & - & $32(24.24 \%)$ \\
$>61$ & - & - & - & 1 & - & 5 & - & $6(4.55 \%)$ \\
Total & $5(3.79 \%)$ & $1(0.76 \%)$ & $7(5.30 \%)$ & $87(65.91 \%)$ & $14(10.60 \%)$ & $17(12.88 \%)$ & $1(0.76 \%)$ & $132(100 \%)$ \\
\hline
\end{tabular}

Abdominal route, 100 patients (75.76\%); vaginal route, 31 patients (23.48\%); and minimal access surgery (MAS), 1 patient (0.76\%)

Table 4: Association of types of hysterectomy with LOHS in days

\begin{tabular}{|c|c|c|c|c|c|c|c|c|c|}
\hline Types & 3 days & 4 days & 5 days & 6 days & 7 days & 8 days & 9 days & $\geq 10$ days & Total \\
\hline $\mathrm{TAH}+\mathrm{USO}$ & 2 & 2 & - & - & 1 & 1 & - & 1 & 7 \\
\hline $\mathrm{TAH}$ & 1 & 1 & 2 & - & - & - & - & 1 & 5 \\
\hline $\mathrm{TAH}+\mathrm{BSO}$ & 16 & 17 & 19 & 13 & 10 & 1 & 1 & 10 & 87 \\
\hline Sub-TAH & - & - & - & - & - & - & - & 1 & 1 \\
\hline $\mathrm{NDVH}$ & 6 & 4 & 2 & - & 1 & 1 & - & - & 14 \\
\hline $\mathrm{VH}+\mathrm{PFR}$ & 3 & 5 & 1 & 1 & 3 & 1 & 1 & 2 & 17 \\
\hline LAVH & - & - & - & 1 & - & - & - & - & 1 \\
\hline Total & $28(21.21 \%)$ & 29 (21.97\%) & 24 (18.18\%) & 15 (11.36\%) & $15(11.36 \%)$ & $4(3.04 \%)$ & $2(1.52 \%)$ & 15 (11.36\%) & 132 \\
\hline
\end{tabular}


Table 5: Distribution of types of hysterectomy with indications

\begin{tabular}{lcrlrlc}
\hline Types & Fibroid & AUB & Prolapse & CPP & Adnexal SOL & Total \\
\hline TAH & 1 & 4 & - & - & - & 5 \\
Sub-TAH & 1 & - & - & - & - & 1 \\
TAH + USO & 2 & 4 & - & 1 & - & 7 \\
TAH + BSO & 30 & 37 & - & 11 & 9 & 87 \\
NDVH & - & 10 & - & 4 & - & 14 \\
VH + PFR & - & - & 17 & - & - & 17 \\
LAVH & - & 1 & - & - & - & 1 \\
Total & 34 & 56 & 17 & 16 & 9 & 132 \\
\hline
\end{tabular}

41 to 50 years age-group as the most common age-group followed by 31 to 40 years age-group. There was a tendency to perform BSO in patients who were above 35 years of age and had completed their family. Sivapragasam et al.' noted 41 to 50 years as the most common age-group undergoing hysterectomy; with a wide age distribution ranging from 32 to 75 years. Saravana et al. ${ }^{2}$ in their study also had 41 to 50 years age-group as the most common. The age range was noted to be wider from 20 to 75 years.

In our study, most of the patients were para 2 and above. Hysterectomies were done in 12 nulliparas who were above 35 years of age. Similar results were noted in the study conducted by Saravana et al. Majority of patients were para 2. Sivapragasam et al. noted most of their cases were para 2 and above; five patients in their study were nulliparas. Palve et al. $^{3}$ in their study noted hysterectomies in para 2 and above. There were no nulliparas in their study. Tendency to perform a hysterectomy in multiparas is noted in all the studies. This may be due to the fact that multiparas have completed family and reproduction is not an issue with multiparas.

In our study, the most common indication of hysterectomy was AUB. We used FIGO PALM-COEIN classification of AUB $\left(P_{3} A_{2} L_{26} M_{8} C_{0} E_{14} I_{0} N_{2}\right)$. Twenty-six patients were having $A U B$ due to fibroid. The second common indication of hysterectomy was due to fibroid (Table 2) presenting as pain and/or lump abdomen. With an increase in age, uterovaginal prolapse as the cause of hysterectomy seems to be common in our analysis. However, histopathological correlation posthysterectomy could not be done. This is a limitation of our study. Sharma et al. ${ }^{4}$ study noted AUB as the commonest cause for hysterectomies, followed by pelvic organ prolapse. AUB was the commonest indication in a study conducted by Medhi et al. $^{5}$ These indications were correlated with histopathological findings. Uterine fibroid and adenomyosis were the commonest histopathological findings. Sivapragasam et al. in their study also had AUB as the commonest indication followed by fibroid.

In present study, $75.76 \%$ (100 patients) underwent hysterectomy by abdominal route, $23.48 \%$ (31 patients) by vaginal route, and $0.76 \%$ (one patient) by MAS (LAVH). The abdominal route is most commonly done as most surgeons in our institute are well versed with it. Limited facilities for MAS were one of the main reasons for its low usage. Prasad et al. study ${ }^{6}$ had $81.8 \%$ hysterectomy by the abdominal route and $18.2 \%$ by vaginal route. Abdominal hysterectomy was also the commonest route followed by vaginal and MAS in a study by Patil et al. ${ }^{7}$ An audit of hysterectomy by Sivapragasam et al. also noted abdominal route as the most common.

The most common indication in the abdominal approach was AUB, that is, 45 out of 100 patients, followed by uterine fibroids
(34 patients). In the vaginal route, $\mathrm{VH}+\mathrm{PFR}$ was done in 17 patients with genital prolapse, and NDVH was done in 10 patients with AUB and 4 patients with CPP. The Cochrane review recommends that vaginal hysterectomy should be performed in preference to abdominal hysterectomy, if possible. ${ }^{8}$ The route of surgery must be individualized to the patient and surgeon's preference. In our study, the abdominal route was the commonest route; this could be mostly related to the surgeon's preference.

In our study, the most noted frequency for LOHS was 4 days, and the mean LOHS was $6.07 \pm 0.376$ days. The longest day of LOHS observed was 29 days; the patient was a case of the previous cesarean with anemia and uterine fibroid: She developed wound gaping during the hospital stay. The LOHS of 15 patients was $\geq 10$ days, and among them, 7 patients were anemic prior to surgery. The LOHS was shorter for all cases who had undergone NDVH with an average of 4.2 days. An audit by Pandey et al. ${ }^{9}$ on LOHS noted $8.7 \pm 2.8$ days, $6.8 \pm 1.6$ days, and $6.1 \pm 2.6$ days for abdominal, vaginal, and laparoscopic approaches, respectively. Leung et al. ${ }^{10}$ in their analysis also noted LOHS as $6.7 \pm 2.5$ days, $4.9 \pm 2.4$ days, and $4.2 \pm 3.4$ days for abdominal, vaginal, and laparoscopic approaches, respectively. MAS definitely has a shorter hospital stay. With limited facility infrastructure, NDVH whenever feasible can have a comparable outcome, along with an added advantage of no scar in the abdomen at all.

Twelve additional surgical procedures (11 appendectomies and 1 cholecystectomy) were performed. A study by Salon et al. ${ }^{11}$ concluded that an incidental appendectomy at the time of benign gynecologic procedures does not increase postoperative complication rates or LOHS, and it further prevents the complications of appendicitis. Nowadays, people are more conscious about their health and they demand for these additional surgical procedures, if possible.

\section{Conclusion}

In settings with limited resources, women usually bear their problems till late and have a firm belief in a permanent cure by hysterectomy due to the lack of awareness of the impact of surgical interventions. Advances in medicine and technology have made conservative management possible nowadays. Various routes of hysterectomy are at hand with its disadvantages and advantages. NDVH is an established safe surgical procedure but such route is lesser used. The reason could be less exposure to such procedures. MAS or robotic surgery is not a feasible option in low-resource settings. Additional surgical procedure is an advantage in the abdominal route, but studies of the plausible risks that may be associated with such procedures are also lacking. The impact of preservation of the ovary is also a lesser-explored area. Hysterectomy seems to be the first option in treatment where resources are limited. Symptomatic relief is palpable: But studies assessing the impact of hysterectomy, for example, psychological, sexual, social, economical, etc., are few. It is time to review our approach to benign conditions before considering surgery as the first option.

\section{Clinical Significance}

In our study, long-term follow-up report is lacking, so such impact could not be analyzed. More prospective data comparing indications of hysterectomy with its impact may be helpful in streamlining absolute indications, and patients may be benefitted 
from avoiding the adverse effects of surgical interventions. This may also help to standardize guidelines for hysterectomy, avoid unnecessary surgical interventions, and consequent adverse effects associated with it.

\section{References}

1. Sivapragasam V, Rengasamy CK, Patil AB. An audit of hysterectomies: indications, complications and clinicopathological analysis of hysterectomy specimens in a tertiary care center. Int J Reprod Contracept Obstet Gynecol 2018;7(9):3689-3694. DOI: 10.18203/23201770.ijrcog20183778.

2. Saravana A, Patil SB, Patil SS. Clinopathological study of hysterectomised specimens. Int J Reprod Contracept Obstet Gynecol 2016;6(1):246-248. DOI: 10.18203/2320-1770.ijrcog20164667.

3. Palve TT, Raghav M. Clinical trends, indications, postoperative complications of hysterectomy patients-a retrospective study. Global J Med Res 2019;19(2):Version 1.0. DOI:10.17406/gimra.

4. Sharma C, Sharma M, Raina R, et al. Gynecological diseases in rural India: a critical appraisal of indications and route of surgery along with histopathology correlation of 922 women undergoing major gynecological surgery. J Mid-Life Health 2014;5(2):55-61. DOI: 10.4103/0976-7800.133988.
5. Medhi P, Dowerah S, Borgohain D. A histopathological audit of hysterectomy: experience at a tertiary care teaching hospital. Int J Contemp Med Res 2016;3(4):1226-1228. DOI:10.21276/ijcmr.

6. Prasad DR, Nair NV. Retrospective analysis of elective hysterectomy cases in a tertiary care centre. Int J Reprod Contracept Obstet Gynecol 2018;7(9):3714-3717. DOI: 10.18203/2320-1770.ijrcog20183782.

7. Patil HA, Patil A, Mahajan SV. Histopathological findings in uterus and cervix of hysterectomy specimens. MVP J Med Sci 2015;2(1):26-29. DOI: 10.18311/mvpjms/2015/v2/i1/793.

8. Nieboer TE, Johnson N, Lethaby A, et al. Surgical approach to hysterectomy for benign gynaecological disease. Cochrane Database Syst Rev 2009;2009(3):CD003677. DOI: 10.1002/14651858. CD003677.pub4.

9. Pandey D, Sehgal K, Saxena A, et al. An audit of indications, complications and justifications of hysterectomy at a tertiary hospital in India. Int J Rep Med 2014;2792273:1-6. DOI: 10.1155/2014/279273.

10. Leung PL, Tsang SW, Yuen PM. An audit on hysterectomy for benign disease in public hospitals in Hong Kong. Hong Kong Med J 2007;13(3):187-193. PMID: 17548906.

11. Salon EM, Schey D, Penalver M, et al. The safety of incidental appendectomy at the time of abdominal hysterectomy. Am J Obstet Gynecol 2003;189(6):1563-1567; discussion 1567-1568. DOI: 10.1016/ s0002-9378(03)00936-0. 